Carnets de géographes

GÉOGRAPHES.

\title{
Créations sonores et émotions : une géographie strasbourgeoise
}

Barbara Morovich et Pauline Desgrandchamp

\section{(2) OpenEdition}

1 Journals

Édition électronique

URL : http://journals.openedition.org/cdg/689

DOI : $10.4000 /$ cdg. 689

ISSN : 2107-7266

Éditeur

UMR 245 - CESSMA

Référence électronique

Barbara Morovich et Pauline Desgrandchamp, «Créations sonores et émotions : une géographie strasbourgeoise », Carnets de géographes [En ligne], 9 | 2016, mis en ligne le 20 décembre 2016, consulté le 19 avril 2019. URL : http://journals.openedition.org/cdg/689; DOI : 10.4000/cdg.689

La revue Carnets de géographes est mise à disposition selon les termes de la Licence Creative Commons Attribution - Pas d'Utilisation Commerciale - Pas de Modification 4.0 International. 
Carnets de géographes, $\mathrm{n}^{\circ} 9$, Juin 2016

Rubrique Carnets de Recherches

\title{
CRÉATIONS SONORES ET ÉMOTIONS : UNE GÉOGRAPHIE STRASBOURGEOISE
}

\author{
BARBARA MOROVICH \\ Laboratoire Amup, ENSAS, Anthropologie urbaine \\ barbaramorovich@yahoo.fr \\ PAULINE DESGRANDCHAMP
}

Laboratoire ACCRA, EA “20, Arts Visuels, spécialité Design contact@pauline-desgrandchamp.com

\begin{abstract}
Résumé
L'article s'intéresse à des émotions spatialisées induites par l'expérience du sonore et veut affiner le propos selon lequel la dimension émotionnelle ne relève pas uniquement d'une sphère individuelle mais plutôt d'une sphère sociale qu'elle peut contribuer à éclaircir. A partir d'études de cas, il s'agit d'admettre que le sonore, à travers les émotions qu'il engendre, participe à fabriquer l'identité des lieux ou à la révéler aux usagers. Si « le son publicise l'espace » (Amphoux, 2014), certains projets sont aussi le signe d'une certaine manière de concevoir la ville de la part des institutions. De quelle manière l'environnement sonore interagit-il avec une identité urbaine que la ville de Strasbourg souhaite véhiculer? Comment des émotions accompagnent-elles ces changements? Si certains quartiers, comme le Neudorf, fer de lance du marketing urbain strasbourgeois, sont mis en avant à travers l'art contemporain, ce qui suscite des émotions controversées, à Hautepierre, quartier populaire en rénovation urbaine, des projets sonores sont portés par des associations pour révéler des mémoires liées au changement urbain.
\end{abstract}

Mots-clés : environnement sonore, émotions, identité, changements urbains, Strasbourg

\begin{abstract}
The article is about emotions induced by the experience of sound. It aims at sharpening the assumption that emotional dimension is not only about an individual sphere, but rather a social sphere, which can contribute to its comprehension. Through case studies, we will argue that sound, through the emotions it engenders, participates in generating specific places identity or revealing it to the users. If " the sound makes the space public " (Amphoux, 2014), some projects are also the sign of a certain way to design the city by the institutions. How the sound interacts with an urban identity that the city of Strasbourg wishes to convey? How feelings accompany these changes? Some neighborhoods, like Neudorf, backbone of the urban marketing of Strasbourg, are put
\end{abstract}


Carnets de géographes, $n^{\circ} 9$, Juin 2016

Rubrique Carnets de Recherches

forward through the contemporary art, arousing controversial emotions; for Hautepierre, popular district in urban renovation, projects with sound are carried by an association to reveal memories connected to the urban changes.

Keywords: soundscape, emotions, identity, urban changes, Strasbourg 


\section{Introduction}

Longtemps ignorée par les sciences sociales, la dimension émotionnelle révèle des liens entre la sphère individuelle et la sphère sociale (Bernard, 2015 ; Crapanzano, 1994). Si les émotions sont produites par le social, selon le sociologue Julien Bernard, elles le produisent aussi. Chaque individu a un rapport affectif, évolutif et spécifique à l'espace qui peut se traduire dans des émotions spatialisées, comprises comme phénomène social. Un certain nombre de travaux, notamment ceux de Denis Martouzet et son équipe (UMR CITERES) (Martouzet, 2007, 201“ , 2014 ; Audas, 2010 ; Feildel, 2010, 201“') ont démontré l'intérêt d'une approche affective de la ville et de ses acteurs. Benoît Feildel (201“) s'interroge sur la prise en compte de la dimension affective en

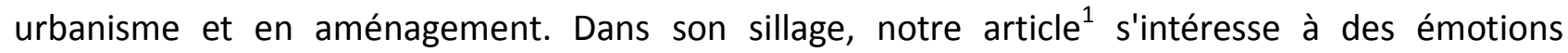
spatialisées en ville, induites plus particulièrement par l'expérience du sonore. Quel est leur rôle dans la création d'une nouvelle identité urbaine?

Selon Solène Marry, "les représentations du sonore sont non seulement des productions individuelles, mais aussi contextuelles et sociétales " (2011: 29). Elles représentent un système d'attitudes qui induisent des pratiques spatiales, à partir d'un certain environnement sonore (2011 : 25, Figure 1). Pour Anderson et Holden (2008), on peut même qualifier d'« affective urbanism » des politiques urbaines d'envergure qui visent à la transformation de certains espaces urbains et qui convoquent, pour reprendre l'expression de Benoît Feildel, un "vocabulaire conceptuel spécifique à la logique des affects et des émotions " (201“). Nous verrons ainsi de quelle manière le sonore et l'émotionnel font partie d'une identité urbaine que la ville de Strasbourg souhaite véhiculer à travers des projets en cours. Nous nous demanderons également comment des émotions en relation avec ces projets sonores accompagnent les changements urbains, qu'il s'agisse d'un processus de gentrification d'un côté ou d'une rénovation urbaine de l'autre. Si certains quartiers, comme le Neudorf, fer de lance des politiques d'embellissement et du marketing urbain strasbourgeois, sont mis en avant à travers une sémantique en lien avec la durabilité et l'art contemporain, pour d'autres, à l'instar des quartiers populaires stigmatisés et en rénovation comme Hautepierre, la sémantique de la mixité cache une entreprise sécuritaire, malgré la tendance à susciter des adhésions à travers le registre affectif et les images de la " participation des habitants" (Morovich, 2012). Ici l'espace public, rénové selon le principe de la prévention situationnelle, se veut moins appropriable notamment par les groupes considérés comme déviants. Comment le sonore est-il interprété par les usagers de ces espaces ? Qui partage une certaine émotion et qui ne la partage pas? Quelles sont les raisons de ces émotions ? Finalement, la ville affective, dont la «ville émotionnelle " fait partie, est-elle aussi une ville inclusive?

\footnotetext{
1 Nous remercions Valérie Lebois, psychosociologue (ENSA-AMUP), et Christophe Gleitz (IEN-Strasbourg) pour leur relecture et leurs conseils qui ont permis d'enrichir cet article. Nos remerciements vont aussi aux personnes nous ayant accordé des entretiens, et plus particulièrement aux membres de l'association Horizome et à Philippe Lepeut. Nous portons toutefois la responsabilité des propos tenus dans cet article.
} 
Figure 1 : Localisation des projets étudiés à Strasbourg

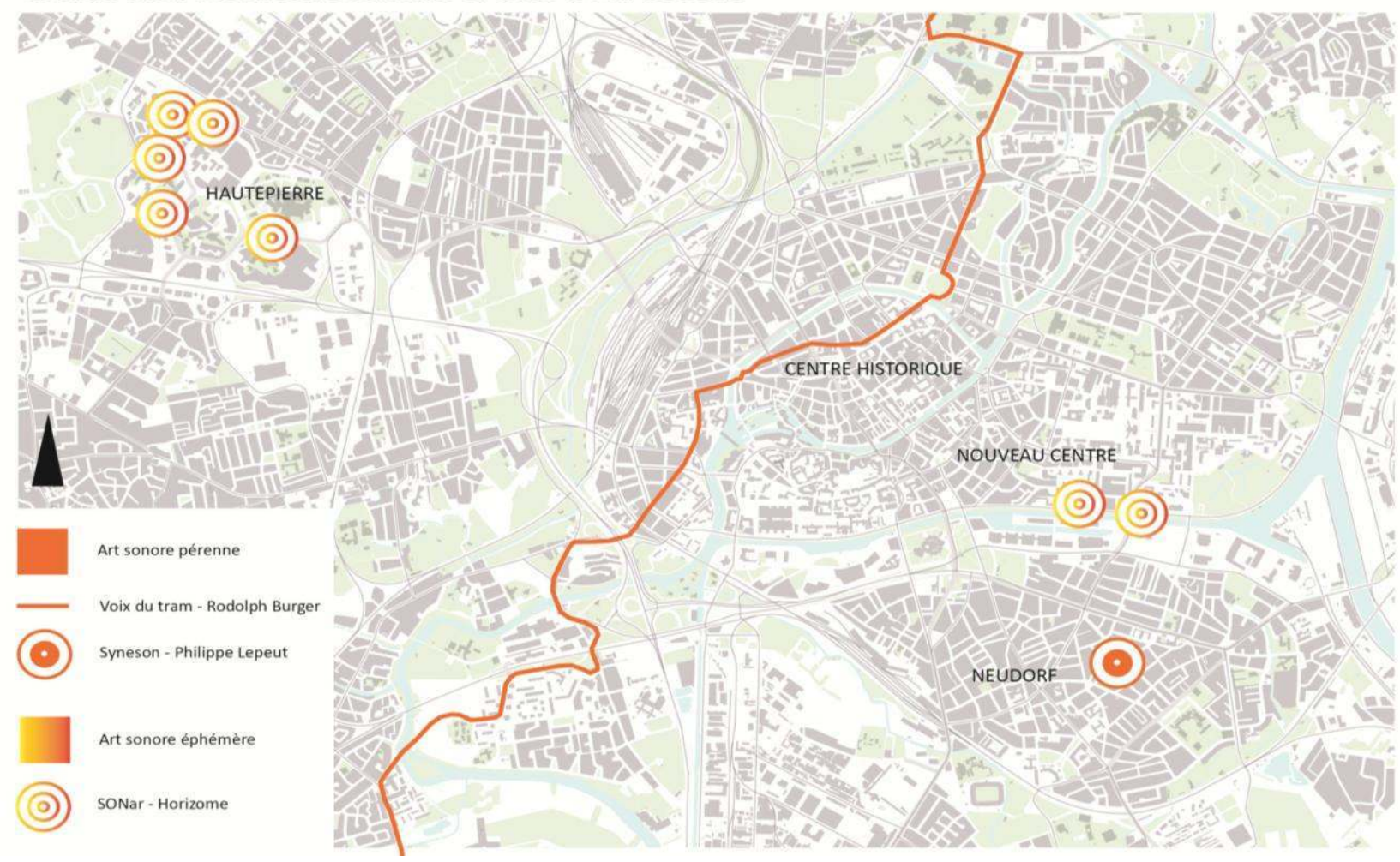

Source : P. Desgrandchamp, 2016.

Pour répondre à ces interrogations, nous nous appuierons sur trois études de cas : deux projets sonores institutionnels (Voix du tram et Syneson), qui concernent surtout des quartiers centraux, et un autre projet sonore, émanant d'une association (SONar), qui peut également nous servir de prétexte pour parler d'un quartier, de ses vécus, de ses mémoires oubliées. Notre principale question de recherche émane de leur comparaison : loin de penser que ces projets s'opposent radicalement les uns aux autres, nous montrerons, à travers l'analyse des émotions qu'ils suscitent, que leurs cadres de production sont imbriqués. Même dans le cas de projets situés dans des espaces plus périphériques subissant a priori moins de pression institutionnelle, les institutions arrivent tout de même à les inspirer et à les guider, au moins partiellement. Si les émotions qu'ils suscitent sont différentes, tous ces projets relèvent du même " urbanisme affectif ».

Pour la méthodologie de notre terrain, mené dans le cadre du projet de recherche en cours « De Strasbourg à Johannesburg: l'art de mettre la ville en mémoire ? La fabrique des mémoires alternatives à travers des dispositifs sonores et numériques »-financé par la Maison des Sciences de l'Homme Paris-Nord - et coordonné par Barbara Morovich, nous avons choisi d'employer plusieurs techniques d'enquête. Un questionnaire a été établi pour le projet sonore du tram (Voix du tram) et posté sur internet. II visait l'analyse des souvenirs liés à la réception du projet et la comparaison avec le présent. La méthode de l'entretien semi-directif et de l'observation des pratiques a été préférée pour l'étude du projet d'art sonore au Neudorf (Syneson), beaucoup plus récent que le premier. Concernant le projet SONar, la méthodologie expérimentale, basée sur la démarche test-erreur nécessitait un questionnaire, mais aussi de l'observation et des entretiens compréhensifs après utilisation ont été mobilisés. Ces méthodologies complémentaires, sur 
lesquelles nous reviendrons plus précisément en présentant les projets, ont permis la triangulation et le croisement des résultats. Concernant le dernier projet, le SONar, notre posture est en outre fortement réflexive, car nous avons participé, à différents moments, à la mise en œuvre des projets de l'association Horizome qui est à l'origine de ce projet et au dialogue, parfois conflictuel, entre les acteurs.

\section{La ville sonore : une question ambigüe ?}

Selon Pascal Amphoux (2014), l'identité sonore d'une ville est une notion complexe : elle est d'abord caractérisée par des sons qui donnent l'impression qu'un lieu reste identique à lui-même (autonomie), ensuite par des sons qui permettent de reconnaître le lieu en tant que "signatures sonores" (reconnaissance), et finalement par des sons "ordinaires et incarnés " auxquels les habitants s'identifient (appartenance). La notion " d'identité » appliquée à la ville a, quant à elle, une connotation politique évidente : I'identité de la ville, ce "discours qui permet de croire le monde ordonné " (Gervais-Lambony, 2004: 470) est composée d'un ensemble d'acteurs et de pratiques, mais est aussi l'expression d'un pouvoir. Selon Nicolas Bautès et Claire Guiu (2010), il s'agit d'observer comment les politiques mobilisent, voire instrumentalisent la culture dans les stratégies urbaines, en lien notamment avec des processus de fort changement. Comment ces derniers, à travers les stratégies identitaires territoriales qu'ils mettent en place, se servent-ils de la culture, et notamment des identités sonores, pour avoir un impact dans des lieux ? Quels rôles les émotions jouent-elles dans ces processus ? Dans ce sens, l'identité sonore de certains lieux de la ville peut se comprendre de manière ambivalente : tant comme processus de marketing urbain, expression qui désigne l'ensemble des opérations lancées par une ville afin de promouvoir son image et son développement, que comme processus d'expériences sensibles, en lien avec la mémoire des lieux et l'appropriation des usagers de la ville. Nous avons déjà souligné, en accord avec Benoît Feildel (200“), que la sphère affective est invoquée dans des projets urbains d'envergure, tant dans le cadre d'une opération de gentrification que dans celui d'une rénovation urbaine. Nous faisons l'hypothèse que des projets sonores, mobilisés à la fois par des institutions et par des associations, revêtent un rôle majeur dans ces contextes de transformation. II s'agit d'interroger ce postulat, en mettant en avant les émotions suscitées par des projets sonores dans la nouvelle Eurométropole strasbourgeoise. Il est à noter que la préoccupation des institutions et municipalités françaises pour le sonore, et plus précisément - comme il est désigné alors - pour le bruit date de la fin des années 1970, époque à laquelle est créé par exemple le Centre d'information et de documentation sur le bruit (CIDB) ${ }^{2}$. Le " bruit ambiant » se comprendrait comme une nuisance. Pourtant, il est plus le résultat d'un jugement sur un son qu'une donnée objective : on pourrait à ce propos évoquer que la maîtrise du bruit et son acculturation ont été une préoccupation du pouvoir politique depuis le Moyen Age (Gutton, 1970). Partant de l'idée que l'environnement sonore fait partie intégrante de l'identité d'un lieu (Murray Schäfer, 2010) et que les bruits ambiants sont des témoins privilégiés des pratiques quotidiennes et des repères singuliers du fait d'habiter la ville (Delage, 1989), il devient primordial de s'intéresser à

\footnotetext{
2 À l'initiative du ministère de l'Environnement, le CIDB « est l'interlocuteur privilégié tant du grand public que des quelque 1000 organismes publics ou privés intervenant dans le domaine de la gestion de l'environnement sonore ». Site : http://www.bruit.fr/le-cidb/qui-sommes-nous-/
} 
l'émergence de la notion d'« ambiance sonore ».

Ce concept à la croisée entre l'analyse de conditions matérielles et d'un " ressenti " sensoriel et affectif, se prête à des usages multiples. Les premières recherches concernant les ambiances et les territoires sonores ont été menées par Jakob von Uexküll au début $d u X X^{\text {ème }}$ siècle : les bruits seraient compris comme "des stimuli perceptibles agissant sur l'homme en fonction de son umwelt ou univers propre " (1965:69). Pour Jean-François Augoyard (1979), I'ambiance sonore en tant que "bruit de fond" facilite l'analyse audio-ethnographique pour déceler les fonctionnements de l'usager vis-à-vis du territoire urbain dans lequel il évolue. Cette notion d'ambiance peut également se comprendre comme un dispositif-outil qui aide à caractériser un lieu. Julien Tardieu (2006) a montré comment la forme sonore peut découler de la fonction. II insiste ainsi, par l'utilisation d'enregistrements de terrain, sur le fait qu'instrumentaliser le bruit de fond peut "re-designer" son ambiance: en d'autres termes, transformer la propre matière du bruit ambiant pour le rendre moins nuisible (Tardieu, 2006). Finalement, une certaine ambiguïté se dégage lorsque l'ambiance, utilisée par les professionnels de la ville à travers ce qu'on peut appeler le "marketing sensoriel », renvoie surtout à une dimension matérielle, subjective et perceptive sans en interroger le sens politique et sociétal (Chadoin, 2010).

La notion de soundscape (paysage sonore), introduite par Murray Schäfer (2010), se révèle également opérationnelle dans l'idée d'un environnement sonore "perçu ». Dans une étude sur le soundscape urbain "idéal " à partir d'une recherche dans trois villes françaises (Paris, Lyon et Nantes), Catherine Guastavino (2006) analyse des sons urbains jugés de manière positive ou négative et apporte des éléments importants pour notre analyse. Après la commande et la réalisation, comment les usagers sont-ils affectés par le sonore en ville ? Dans quelle mesure les émotions suscitées par des projets sonores sont-elles, ou non, le signe de l'appropriation de certains projets urbains ? Comment le sonore favorise-t-il l'appropriation ? Notre hypothèse est que ces projets sonores ont un rôle d'acculturation: ils permettraient l'identification, la reconnaissance et l'acceptation d'un nouveau lieu. Ils contribueraient donc à véhiculer une identité urbaine institutionnelle, bien qu'en constante formation. Parce que la Ville de Strasbourg est pionnière dans l'affirmation d'un art public sonore, elle constitue une étude de cas privilégiée. De quelle manière des associations, des collectifs et des artistes font-ils écho à cette volonté politique ? Quel est le ressenti des habitants de la ville au devenir de ces projets ?

Le compositeur Albert Mayr développe l'idée que le paysage sonore, en tant que phénomène naturel, agit sur l'auditeur en fonction à la fois de sa perception physiologique et de sa représentation esthétique : "Le paysage sonore donc, et on accepte aujourd'hui cette idée pour le paysage visuel également, est une construction subjective et changeante qui varie de sujet en sujet, en fonction de son background culturel et social particulier " (2012 : " 4). II sera alors intéressant de déceler également les dissonances entre des façons de comprendre le territoire du point de vue des émotions sonores: d'une part lorsqu'on analyse des projets sonores institutionnels permanents, d'autre part en examinant le caractère éphémère d'autres projets, plus tournés vers la reconnaissance mémorielle des habitants. 


\section{Des projets sonores innovants à Strasbourg}

A Strasbourg, des installations sonores contribuant à créer à la fois des ambiances et un nouveau décor urbain sont le fruit des politiques d'aménagement et concernent au moins deux aspects : la mobilité représentée par le tram, pièce maîtresse de projets urbains et qui participe à une nouvelle image de la ville, et le renouvellement urbain de certains quartiers à grand enjeu urbain (rénovation ou gentrification). Ces projets sonores institutionnels ont la caractéristique d'être peu négociés avec les habitants, et suscitent des réactions différentes auprès des usagers, y compris pour le caractère novateur du sonore : comment sont-ils dès lors acceptés ?

\section{Les « Voix du tram » : un projet sonore identitaire}

Selon Jean Orselli (2004: 217, cité par Redondo, 2012 : “), le tram s'apparente à " un discours » inscrit au cœur des politiques urbaines. Objet fonctionnel et fictionnel, son message est fédérateur car il est perçu comme un "composant urbain indissociable de l'identité des villes " (Redondo, 2012 : “). Cette portée fictionnelle fait que le tram est le réceptacle de plusieurs projets culturels et artistiques, à travers lesquels il singularise à la fois les sites touchés et le trajet lui-même.

Dès 2000, lors de la création de la deuxième ligne de tramway de la Compagnie des Transports Strasbourgeois (CTS), la Ville de Strasbourg, alors dirigée par la gauche, décide d'installer dans les rames une œuvre sonore, les Voix du tram, du musicien Rodolphe Burger. Cette commande publique s'inscrit dans une volonté de déployer l'art contemporain dans l'espace urbain strasbourgeois, notamment tout au long du tracé de la ligne B (Dernières Nouvelles d'Alsace DNA, "Les voix du tram ", 19 Décembre 2001). Ainsi, plusieurs œuvres artistiques sont prévues et vont jalonner les arrêts du tram, ce qui coïncide d'ailleurs avec l'ouverture du Musée d'art moderne et contemporain de Strasbourg, inauguré en 1998. L'art est mis au service de l'usager afin de le sensibiliser à la création contemporaine". On peut d'ailleurs souligner que l'art dans l'espace public est un outil de régénération urbaine utilisé de plus en plus par des politiques publiques afin d'asseoir la transformation des espaces urbains (Guinard, 2010, Vivant, 2009).

Dans le cadre d'un appel à participation citoyenne, Rodolphe Burger a enregistré puis traité les voix de citoyens comme des motifs sonores, afin d'identifier chacun des arrêts de tramway et de donner à entendre la diversité sociale de Strasbourg. L'artiste souhaitait provoquer un effet de décrochement, une invitation au voyage. Ce projet social et impliqué, dans le sens où l'auteur a proposé un usage encore non conventionnel des voix des habitants, envisage de mettre en abîme les différentes identités strasbourgeoises de manière territorialisée, ce qui a parfois comme effet l'assignation des sujets interviewés à des espaces précis. C'est en insistant sur une classification d'accent en fonction des quartiers traversés, que ce projet relie un jeune homme criant "Krimmeri, stade de la Meinau " au quartier populaire de la Meinau, et un historien expliquant que place Broglie se prononce également « Breuille », à la place historique éponyme.

Le projet, toujours en activité, reste exemplaire dans sa démarche, même si le travail sonore, jugé

\footnotetext{
C'est d'ailleurs dans ce but que le CEAAC (http://ceaac.org/histoire-du-ceaac) avait vu le jour en 1987 : « Le CEAAC (Centre Européen d'Actions Artistiques Contemporaines) est né sous l'impulsion de la Région Alsace, du Conseil Général du Bas-Rhin et de la Ville de Strasbourg dans le but de démocratiser l'art contemporain auprès d'un large public et de soutenir la création plastique des artistes régionaux ». Son Président et fondateur est l'homme politique Robert Grossmann (ex-UMP).
} 
immatériel et non-plastique n'avait pas été considéré comme une "œuvre d'art » par la municipalité ${ }^{4}$. Il paraît donc intéressant de comparer le ressenti actuel des usagers par rapport au souvenir de la réception initiale de ce projet. L'hypothèse est que le projet a favorisé une appropriation du tram et le renforcement d'une identité strasbourgeoise. Pour cela, un court questionnaire semi-directif a été envoyé sur internet (messagerie électronique, Facebook). Les questions suivantes étaient posées : "Étiez-vous au courant que ces annonces de station émanent d'un projet d'art public ?", "Qu'a suscité votre première écoute du projet sonore "Voix du tram" ?", "Avez-vous reconnu différents types d'accent et de voix ?", "Pourriez-vous citer au moins une de ces voix?", "Comment trouvez-vous ce projet aujourd'hui ?", "Avez-vous autre chose à ajouter?" ".

Sur un échantillon de 47 personnes, hommes et femmes, entre 20 et 67 ans, une seule réponse était négative à la question: "Qu'a suscité votre première écoute du projet sonore "Voix du tram" ? ". Les réponses données permettent donc d'attribuer une valeur positive au tram à travers le son : dans son étude, Guastavino (2006) montre déjà que les bruits des transports en commun sont largement acceptés. Le projet artistique Voix du tram facilite ultérieurement l'acceptation, en ajoutant une portée identitaire qui est, à notre avis, "cumulative " : tournée vers le renouveau et l'international, elle exprime également un attachement à l'identité alsacienne. Les interviewés se rappellent leur première réaction qui est, le plus souvent, liée à la surprise et l'amusement. A ceci s'ajoute la reconnaissance de la pluralité des voix, des accents, notamment l'alsacien : " II y a des voix d'enfants, adultes avec des accents alsaciens et anglais il me semble ", "Oui, il y a l'accent alsacien, russe, une voix type rock... ", "Je croyais toujours que c'était Monica Bellucci, en tout cas c'était la voix d'une femme italienne ", " "Alsass...", puis un accent américain ", etc. Pour autant, les usagers qui vivent à Strasbourg, au bout de quelques années d'utilisation du tram, ne font plus forcément attention aux variations ni aux types d'accent. La majorité des interviewés trouve qu'il faudrait remettre le projet au goût du jour, varier plus, introduire d'autres langues, etc.

Toutefois ce projet reste tout d'abord "familier». Les Voix du tram par les émotions qu'elles suscitent (amusement, surprise), permettent également de passer d'un registre individuel à un autre, plus collectif : ces phrases introduisent du lien entre les usagers qui échangent regards et sourires, et guettent le " premier utilisateur » et sa réaction étonnée. Le projet a fait parler aussi les gens en dehors du tram, des personnes interviewées affirment notamment qu'elles se sont posées des questions sur la relation entre les voix et les lieux, les voix et les sons qui les accompagnent. Beaucoup ont le souvenir de certaines voix, très identitaires, comme des marqueurs, d'autres s'amusent à croire qu'il s'agit des personnages connus et cherchent à deviner lesquels. Attachés aux Voix du tram, beaucoup admettent qu'elles singularisent leur rapport à la ville et font désormais partie intégrante du paysage sonore de Strasbourg. C'est une "routine qui plait » (comme l'affirme un des interviewés), même si l'étonnement n'est plus là et on imagine facilement que les seuls étonnés sont désormais les touristes ou les visiteurs. Le projet Voix $d u$ tram participe donc, à travers les émotions qu'il suscite, de l'acceptation de ce moyen de transport par ses usagers et de cette identité complexe, souhaitée par la municipalité strasbourgeoise. Le sonore, temporel dans sa durée et aléatoire en tant que bruit ambiant (Leipp, 1977), facilite ainsi une reconfiguration constante de sa propre image mentale. Même si l'on note une volonté de

\footnotetext{
4 Selon une chargée de mission pour la Direction de la Culture, ce travail n'a pas été susceptible d'être défini comme « œuvre artistique ». Entretien de P. Desgrandchamp, 10/05/201“.
} 
composer une articulation entre le paysage visuel urbain en mouvement (permis par les cadres des grandes baies vitrées) et les annonces " racontées " des stations, ces Voix du tram se figent dans le temps, à l'instar d'une image visuelle. Mais le consensus généré autour du projet sonore dans le tram, cette surprise amusée, est un élément de plus de son acceptation.

\section{L'art sonore en débat : les émotions du Syneson}

Une autre œuvre sonore strasbourgeoise, plus récente, le Syneson, connaît un destin différent. En 2012, dans le cadre de la rénovation de la place du Marché du Neudorf, quartier de Strasbourg fortement touché par la gentrification, une nouvelle commande publique est lancée pour l'élaboration d'une œuvre artistique. C'est la proposition d'une installation sonore de l'artiste Philippe Lepeut qui remporte l'appel.

Le quartier strasbourgeois du Neudorf est concerné par une fracture qui s'amplifie entre sa partie est, économiquement plus favorisée, et sa partie ouest, plus populaire (Adeus, 201“). Afin d'assoir une nouvelle image et d'insuffler de la " mixité sociale et fonctionnelle " comme l'affirme le Maire de Strasbourg lors de l'inauguration de la place (DNA, "Un cœur à prendre », 1 décembre 201“), la municipalité décide de rénover la place du marché, souhaitant faire de ce lieu le "nouveau cœur $d u$ quartier ". Encore une fois, c'est à l'art sonore qu'on donne le rôle singulier de participer au changement de la place, réaménagée par des paysagistes. C'est une œuvre audacieuse qui est choisie, et c'est autour de cette œuvre que les émotions les plus contrastées se manifestent avant, pendant et après sa mise en place.

Quelques articles de Rue 89 Strasbourg et des DNA décrivent un tiraillement entre des émotions individuelles exacerbées, globalement de rejet du projet, et la volonté pédagogique de l'artiste (DNA, "Opération pédagogie ", 14 Juillet 201"). Dans le même article, les réactions des habitants sont rapportées par les journalistes lors de l'explication publique de l'artiste: «Eh ben, 90000 euros pour entendre l'orage... ", "On a tout gagné ! Les gazouillis d'oiseaux vont attirer tous les chats errants du quartier!" ou encore "C'est quoi ce bruit? Un court-circuit dans les enceintes? ". Nous pouvons d'ailleurs remarquer l'utilisation du terme " bruit " par les usagers, ce qui traduit leur dépréciation du son proposé par l'artiste. En effet, l'art sonore non éphémère par sa rareté en France et par son intrusion dans l'espace provoque des réactions fortes. Toutefois, selon Solène Marry, les réinterprétations des ambiances sonores ordinaires par des artistes ont comme conséquence leur sublimation (2011: 4").

Pour explorer ce processus depuis le début et analyser les ressentis des divers acteurs impliqués, nous avons opté pour la méthode de l'entretien et de l'observation des pratiques, en parallèle à l'analyse des articles de la presse locale. Le but était de suivre ce processus récent, et de renforcer nos hypothèses quant au rôle et à l'importance du sonore dans l'espace urbain.

Selon Philippe Lepeut, l'artiste concepteur ${ }^{5}$ :

Mon idée était de créer un lien sonore en m'appuyant sur les éléments déjà présents et à venir : la loggia de la Médiathèque, la façade du Taps et la pergola. [...] Le dispositif se déclenche en début de chaque heure de 8 heures à 21 heures et diffuse du son pendant une durée variant de 6 à 12 minutes. [...] les problèmes ont concerné la concurrence entre les vrais " oiseaux 》 et les oiseaux enregistrés. Cette friction entre le vrai et le faux, la chose et sa représentation était un des enjeux,

5 Philippe Lepeut, entretien de B. Morovich, 17/09/2015. 
mais cela a été difficilement accepté. J'ai travaillé avec la LPO (Ligue Protectrice des Oiseaux) et fait en sorte d'éviter les conflits avec les oiseaux, les vrais, "les résidents ». [...] Mais finalement mon œuvre, et généralement les œuvres dans l'espace public, a été un prétexte à une opposition politique puisque son installation s'est faite peu de temps avant les élections municipales. L'association "Les amis du vieux Neudorf ", très virulente au départ, semble avoir changé d'avis.

C'est en effet sous fond politique que les polémiques sont orchestrées dans une période préélectorale, notamment par l'association citée, dont la présidente est proche de l'opposition de droite. Et c'est sur l'œuvre sonore que se cristallise notamment cette dispute, tandis que les autres aménagements de la place sont mieux acceptés par les habitants. Toutefois, même du côté des élus de l'opposition, un certain désaccord est présent: pour le Syneson, une démarche d'information avait été menée depuis le départ, ce qui n'est pas le cas pour des œuvres plastiques strasbourgeoises plus couteuses, comme le souligne Robert Grossmann, élu de l'opposition et féru d'art contemporain ${ }^{6}$. L'œuvre sonore, par sa nouveauté et son label " art contemporain ", incarne des émotions contraires et des nombreuses polémiques dans les commentaires des articles cités et au sein de la droite strasbourgeoise: pour ou contre l'art contemporain? Pour ou contre le sonore ? En outre, il semble qu'on ne puisse pas parler de véritable « concertation », puisque selon les habitants et notamment $\mathrm{CS}^{7}$ :

L'œuvre a été imposée par la municipalité, aucune concertation n'a été demandée. Le projet a été présenté comme un état de fait au Conseil du quartier. Lors de la présentation du projet, les habitants étaient sceptiques sur l'œuvre, notamment sur le fait qu'elle prétendait réinjecter des chants d'oiseaux dans un endroit où, par ailleurs, de grands arbres avaient été coupés. Le prix de l'œuvre avait également suscité l'opposition des habitants, alors que le Conseil du quartier demande des choses qui ne sont pas octroyées à cause de l'argent, comme plus de bancs, une fontaine... Le projet, dans un premiers temps, a été largement critiqué, et personne n'était pour. Maintenant je trouve que ce projet n'a pas beaucoup de sens dans le lieu où il est : le trafic de la rue qui passe à côté de la place est intense et ne permet pas de bien entendre les sons.

Les usagers sont partagés et soulignent surtout l'absence de sons bien audibles : "Le dispositif ne fonctionne que de temps en temps...", "Je n'ai jamais entendu aucun son de cette ceuvre sonore... ", "Ils auraient mieux fait d'installer un jeu pour enfant supplémentaire... ", "Quand ça marche, c'est pas mal...", "On ne comprend pas si ça s'active automatiquement .... " (DNA, "Cinquante nuances de silence », 15 février 2015). Mais les polémiques s'adoucissent progressivement: l'acceptation du Syneson semble se profiler et une pétition concerne non pas l'œuvre, mais son fonctionnement (DNA « Un silence qui fait parler », 15 février 2015), preuve que I'habitude au sonore calme les polémiques et que l'appropriation de l'art contemporain se fait progressivement dans ce quartier en voie de gentrification.

Dans les deux cas présentés, les méthodologies différentes permettent d'arriver au même constat général, qui se trouve donc conforté : l'art sonore en particulier contemporain, notamment par les émotions qu'il suscite (aversion et colère pour le deuxième cas, amusement et surprise pour le premier), participe à refonder l'identité d'un lieu en lien avec le changement urbain et une forme de marketing de la ville. A ce titre, on peut être d'accord avec l'affirmation de Pascal Amphoux

6 II s'est félicité de la « démarche mise en œuvre » pour le projet sonore. À l'inverse, il a regretté que la sculpture « Point de convergence » inaugurée place d'Austerlitz en octobre 2012 soit choisie par un conservateur et non pas par un jury. De plus, l'élu souligne la différence de prix (DNA, " L'arbre sonore qui cache la forêt », 2" Octobre 2012). 7 CS, habitante du Neudorf qui était dans le Conseil du quartier à l'époque. Entretien de B. Morovich, 19/09/2015. 
(2014) que le son publicise l'espace: il le révèle et le construit dans sa qualité "publique ». Finalement, le sentiment d'acceptation de cet art sonore passe par un travail de " sensibilisation ». Des ateliers en relation au Syneson sont actuellement menés par la Ville de Strasbourg et le Centre Européen d'Actions Artistiques Contemporaines (CEEAC), afin de faciliter une appréhension de l'écoute quotidienne de la ville. Marketing urbain, gentrification et éveil à l'écoute iraient-ils alors de pair ? L'exemple cité nous permet d'insister sur la prégnance de l'urbanisme affectif défini en amont. Cas non isolé, ce nouveau paradigme d'une "ville sonore vitrine " incarne la quête de compétitivité entre les différentes collectivités territoriales. Comme l'explique Juliette Volcler :

L'installation sonore est en effet en train de se tailler une place dans les politiques d'aménagement des collectivités locales, soucieuses de marketing territorial [...] La ville se fait décor, lieu d'une flânerie organisée, territoire débarrassé, en surface, des conflits sociaux qui le constituent et le transforme. (2014)

La ville sonore favoriserait donc la création de nouvelles coupures socio-spatiales et culturelles, tout en les dissimulant. Qu'en est-il de projets sonores dans un quartier plus périphérique de Strasbourg et des émotions de leurs usagers ?

\section{Territorialiser les émotions sonores}

Depuis les années 1960, des artistes ont manifesté leur intérêt envers les espaces de la ville à travers des parcours urbains sonores (Faubert, 2012). Pour ces créateurs, la relation avec un espace géographique précis et ses habitants est particulièrement significative : les interventions artistiques relèvent toutes d'une articulation complexe entre le territoire, le contenu sonore de la proposition et le corps sensible et affectif du promeneur (Faubert, $2012: 4{ }^{\prime \prime 2}$ ). Julie Faubert met en avant le rapport privilégié de l'artiste sonore avec un lieu, son " corps à corps " avec les espaces. C'est à travers une inscription forte dans le territoire et ses usagers et une implication des artistes, des chercheurs et/ou membres associatifs que certains projets sonores sont menés à Strasbourg, par exemple sur le Campus Universitaire du quartier de l'Esplanade mais aussi par des enseignants d'école d'architecture et des institutions culturelles sur les "Fronts du Neudorf " ${ }^{8}$. Le choix de ces quartiers va de pair avec une demande, de la part de la municipalité, d'y fabriquer une nouvelle identité, en lien avec des changements urbains d'envergure.

Dans ces projets, les bruits ambiants sont enregistrés et écoutés comme des traces mémorielles, provoquant une pluralité d'émotions interprétées par les individus. Parallèlement, dans ces contextes, une "éducation à l'écoute " est proposée, afin d'appréhender de manière analytique les sons pour faire "expérience de l'expérimentation » (Saladin, 2015). Des nouveaux métiers, des nouvelles spécialisations surgissent alors autour de la médiation à l'écoute, et ceci souvent en lien avec le numérique ou l'architecture. Dans un entretien, l'ingénieur du son Daniel Deshayes explique que :

Dans un groupe réuni qui écoute la même chose, il y aura autant d'expériences mnésiques que

\footnotetext{
8 Atelier "Les bruits racontent l'Esplanade ", dans le cadre des Ateliers Culturels et en partenariat avec Radio En Construction, mené entre 2011 et 201“ (P. Desgrandchamp). Journée d'étude interdisciplinaire " De l'exploration sensible de l'espace à la démarche de projet ", co-organisée par l'ENSAS (Valérie Lebois et Dominique Laburte) et I'ACCRA (P. Desgrandchamp.)
} 
d'individus. Chacun s'approprie le son de manière extrêmement profonde parce que cela réfère à notre expérience privée... et, en même temps, collective, car si tel son peut représenter pour tout le monde une même chose, on n'en a pas tous la même expérience. (2015 : 11)

La dimension sonore nous renseigne sur le territoire dans lequel on évolue (Von Uexküll, 1965) ; s'intéresser à son écoute c'est aussi comprendre les différents vécus des groupes, facilitant notamment la mise en récit de leurs mémoires.

Afin de mettre en avant des récits d'usagers de l'espace public dans le quartier populaire de Hautepierre, un projet sonore, le SONar, est conçu au sein de l'association Horizome. Centrée entre autres sur les usages numériques, cette association déploie son action sur la question mémorielle afin de mettre en avant et de valoriser des aspects méconnus de Hautepierre, et notamment des vécus des groupes, habitants et associatifs (Morovich, 2014, 2015). Selon Benoît Feildel (201*), la thématique relative à la mémoire et/ou à la communauté est partie prenante des dimensions contemporaines liées à l'affectivité. On verra comment dans le contexte étudié, le sonore est également partie prenante de celles qu'on peut appeler des affectivités mémorielles.

Hautepierre est un quartier populaire en rénovation urbaine depuis 2009. Quartier stigmatisé et dégradé par l'absence de réhabilitations régulières mais globalement apprécié par ses habitants, il est touché par une rénovation urbaine à visée sécuritaire qui prône l'ouverture de ses " mailles ", des espaces autrefois piétons, une résidentialisation et la " mixité sociale " (Morovich, 2012). Le travail mémoriel d'Horizome a aussi une résonance forte avec les enjeux de la politique de la ville qui propose à des artistes, lors des démolitions-reconstructions, de mener un travail sur les vécus et les mémoires (Veschambre, 2008 ; Morovich, 2014).

\section{Figure 2 : Expérience SONar à Hautepierre, place Jean Giono}

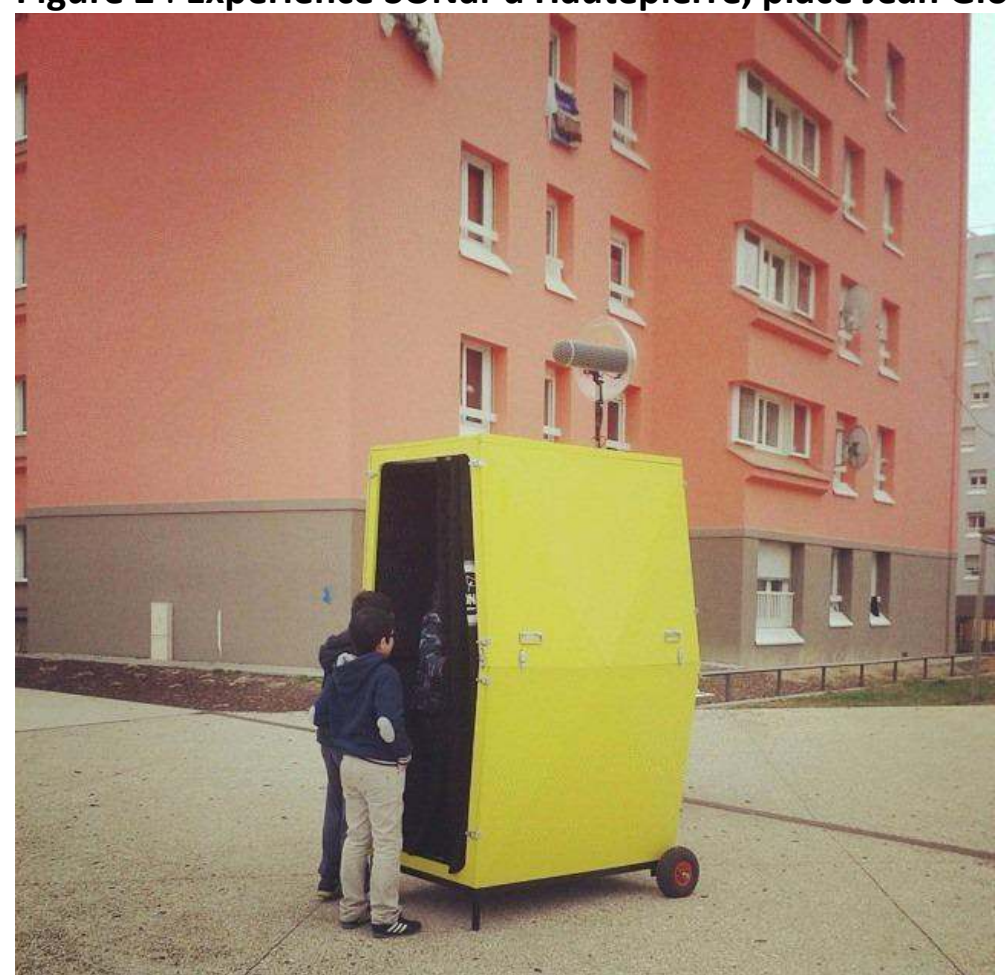

Cliché : P. Desgrandchamp, mars 2015. 
Le SONar ${ }^{9}$ est une cabine mobile de captations sonores et de témoignages sensibles qui permet à l'expérimentateur de (re)découvrir son quotidien et de capturer des sons ambiants. Un dispositif numérique à l'intérieur de la cabine favorise la superposition de commentaires et interprétations des sons ambiants, sur la base d'un questionnaire semi-directif diffusé sur l'interface. L'expérimentateur, par son écoute accentuée et par le biais d'une méthodologie qui agit comme "générateur d'identité sonore urbaine " (Desgrandchamp, 2014) va comprendre le fonctionnement d'un paysage sonore en essayant de noter ce qui se joue dans l'espace (tonalité du son, variation, résonance, couleur, etc.). Cet objet sert aussi à récolter des traces et mémoires de ce qui se joue dans les quartiers en rénovation, à travers un questionnaire semi-directif. Ce dernier porte sur les questions suivantes: "Quels sons entendez-vous ici ? ", "Quels sons ont changé depuis la rénovation de la maille ?", "Quels éléments de ce lieu ont disparu ?", "Comment définir les sons de ce lieu avant la rénovation?", "Les sons sont-ils mieux maintenant ou étaientils mieux avant ? Pourquoi ?".

La méthode choisie propose à la fois une expérience de sensibilisation et d'éveil à l'écoute et un espace de témoignages sur les différentes mutations urbaines de Hautepierre. Ce dispositif est conçu pour participer à la récolte de récits et mémoires alternatives et ainsi nourrir les hypothèses du projet de recherche déjà évoqué. Favorisant l'écoute de son quotidien urbain, il s'agit de prendre conscience de la dimension sensible du sonore qui permet d'identifier les différents espaces de Hautepierre, à travers les paroles des habitants relatant la vie du quartier. C'est une manière de faciliter une écoute active tout en assumant le paradoxe de l'idée de s'enfermer dans une cabine pour prendre conscience de ce qui existe autour de soi. Le SONar, est aussi un objet plastique et mobile, d'un jaune vif, imposant et sur roues. A la différence des autres projets de l'association Horizome, cet objet a été conçu en lien avec des institutions culturelles strasbourgeoises pour répondre à un appel à projet émanant d'un service de l'Eurometropole. De plus, l'utilisation du SONar est conçu dans le cadre des activités du Shadok, la "fabrique du numérique " de la ville de Strasbourg, et en partenariat avec le réseau des médiathèques de I'Eurometropole. A travers ce projet, des acteurs associatifs et institutionnels participent à un projet commun de médiation et d'éveil à l'écoute qui correspond aussi à un projet de thèse en cours $^{10}$.

Le SONar est déployé par l'association Horizome à Hautepierre, et aussi au sein de la presqu'île Malraux, qui fait partie des Fronts du Neudorf. La comparaison des expériences dans ces deux quartiers en lien avec le même projet sonore se révèle particulièrement instructive. La presqu'île Malraux est comprise dans le projet Deux Rives Strasbourg-Kehl, vanté par la municipalité comme "le plus grand projet urbain mené depuis la construction de la Neustadt entre 1871 et 1918 " comme on peut lire sur le site de la Ville de Strasbourg. Si la rénovation de Hautepierre a un aspect sécuritaire certain, notamment dans l'approche de l'espace public, et vise à changer l'image d'un quartier populaire stigmatisé le rendant attrayant pour des populations moins stigmatisées (Morovich, 2012), le projet Deux Rives est une véritable vitrine de projets urbains ${ }^{11}$ et entend

9 Projet co-conçu par P. Desgrandchamp et G. Zabé, lauréat de l'appel à projet SCAN 201", Eurometropole.

10 Thèse de P. Desgrandchamp "Narrativité et plasticité du fait sonore dans une approche Design " au laboratoire ACCRA de Strasbourg, en contrat CIFRE au Shadok pour la Ville de Strasbourg, au sein du projet S.P.H.E.R.E. (Sonorama Participatif des Histoires Extra-ordinaires de nos Rues et de nos Espaces).

11 Sur les "Fronts de Neudorf ", plusieurs infrastructures ont été créés dans les années 2000 : un multiplex UGC (2000), le Vaisseau (Musée scientifique) (2005), la Cité de la musique et de la danse (2006), le grand centre commercial 
dynamiser fortement l'axe vers l'Est et sa frontière avec l'Allemagne. La médiathèque Malraux, maison-mère du réseau des médiathèques de l'Eurometropole, ainsi que le Shadok, régie directe ouverte en 2015, en deviennent les emblèmes culturels. Sur son site, le Shadok ${ }^{12}$ se définit entre autres comme une "vitrine pour les initiatives innovantes des entrepreneurs et créatifs strasbourgeois ". Strasbourg, à travers ces dernières infrastructures et l'impact qu'elles ont sur l'espace par leurs activités et leurs images se présente donc comme une "ville créative " (Vivant, 2009), se servant d'un " urbanisme affectif " (Anderson et Holden, 2008) pour attirer des touristes et des nouveaux habitants dans des quartiers rénovés.

A Hautepierre comme sur la Presqu'île Malraux, le SONar sert à évaluer de l'impact de ces projets mais aussi de l'effet de l'objet sur les usagers. Ici, on n'est pas face à un dispositif qui émet du son, mais au contraire qui le capte. Si l'objet est le même, il sert, dans les deux sites, à des fins différentes : dans le quartier de Hautepierre, le SONar est employé dans un projet associatif pour donner voix à des mémoires en relation au changement urbain. Sur la presqu'île Malraux, il est utilisé dans le cadre d'un partenariat avec des institutions (Shadok et médiathèque) et de festivals numériques locaux pour animer les lieux et participer à les rendre attractifs.

En ce qui concerne la presqu'île, le SONar est utilisé par des enfants mais également par beaucoup d'adultes qui se prêtent au jeu. Le groupe expérimentateur le plus nombreux est constitué d'hommes entre 20 et " 0 ans, portés sur les arts numériques qui n'ont pas de difficulté à utiliser le SONar. Ce sentiment d'être à l'aise conforte alors leur présence sur un site consacré au numérique et ne provoque pas de décalage entre leur ressenti et le lieu.

Dans le quartier de Hautepierre, ce sont surtout les enfants (une trentaine jusque-là) qui sont les utilisateurs du SONar. A la différence de la presqu'île, où l'objet peut rentrer facilement dans le cadre d'un festival numérique, à Hautepierre le SONar interpelle fortement le passant en tant qu'objet: curieux et décalé, il pousse à des interrogations sur son usage, permettant des nombreux échanges, mais cristallise aussi la discussion sur d'autres sujets. Pour les enfants, le SONar est un objet ludique, et ils en détournent bien souvent l'usage. En revanche les adultes prennent les instructions à la lettre, sont plutôt repoussés par l'aspect normatif du questionnaire et, peut-être aussi, par une certaine fracture socioculturelle par rapport au numérique. Un contraste saisissant peut se remarquer entre la faible prise de parole des adultes à l'intérieur du SONar et les discussions et les échanges en dehors de la cabine. Dans les témoignages des adultes sur les changements urbains, le SONar ne semble pas libérer la parole : ils identifient très peu les mutations de la place, malgré des changements majeurs, certains oublient même qu'avant c'était un espace piéton. C'est notamment le "bruit des enfants » qui apparaît en continuité avec la place avant rénovation. Ce manque de reconnaissance du changement peut tenir au fait que les adultes ne sont pas les usagers les plus impliqués dans cet espace, mais nous sommes inclines à croire que si le changement urbain a un impact sur la perception de l'espace, le sonore a ici un rôle mémoriel certain, réactivant des souvenirs. Pour les enfants (7-10 ans), le changement urbain est majeur, en qualité d'usagers principaux de la place, avant et après rénovation. II se dégage un sentiment de satisfaction par rapport à la nouvelle place qui leur octroie plus d'espace de jeu, et un sol bétonné

Rivetoile (2008), la Médiathèque André Malraux (2008), les Archives départementales et celles communautaires. Un Eco-quartier (Danube) dont on met en avant la démarche exemplaire, ainsi que des nombreux immeubles d'habitation et de bureaux sont en construction.

12 http://www.shadok.strasbourg.eu/ 
qu'ils préfèrent au « sable " d'avant: " C'est mieux maintenant » est la réponse la plus fréquente, car " il n'y a plus de sable ». Quelques témoignages d'adolescents se projettent, quant à eux, dans la nostalgie de la place comme elle était avant, se rappelant, lors des entretiens, surtout des aménagements détruits dont les enfants ont peu de souvenirs : "Je me rappelle de tout à peu près : la piste de skate, l'araignée, la tortue ... ", "Ils ont enlevé tous nos souvenirs, les pentes... ", "Je ne sais plus ça c'est où... ". Mais, de manière générale, tous âges confondus, les personnes interviewées admettent que les changements d'ambiance sonore ne sont pas très importants.

Malgré ce qu'on aurait pu croire, car des travaux majeurs ont changé le visage de l'espace, les habitants n'expriment donc ni étonnement, ni protestation. Ils signalent que les sons qu'ils entendent à travers le SONar et qui sont reconnus comme "identitaires", restent les mêmes qu'avant la rénovation. Les " bruits de voitures", est une signature sonore qui revient plusieurs fois dans les entretiens, et ceci est étonnant : lorsque la place était piétonne, deux ans auparavant, il n'y avait que peu (ou pas) de voitures dans ce lieu. Le sentiment de satisfaction quant au " changement » de la place est donc à nuancer. Par ailleurs, l'importance attribuée, dans le passé comme dans le présent, à des bruits en lien avec les voitures et les scooters - des signatures sonores négatives (Guastavino, 2006) invite à croire à la permanence de cette place au sein d'un imaginaire stigmatisé.

\section{Conclusion}

Dans cet article nous avons mis en rapport des émotions provoquées par le sonore avec des changements urbains. Si les émotions qu'ils suscitent sont différentes, tous ces projets relèvent du même " urbanisme affectif " que la ville de Strasbourg souhaite véhiculer. Les exemples analysés, permettent de reconnaitre au son le caractère "d'objet transitionnel », sur lequel, comme l'affirme Solène Marry "le sujet percevant peut agir ou bien comme un signal avec lequel une relation s'établit et qui est maîtrisable ou encore comme un phénomène sur lequel il est impossible d'agir et qui crée un sentiment d'impuissance » (2011:28).

À travers l'analyse de trois projets sonores à Strasbourg, nous pouvons affirmer que les émotions permettent de créer ou d'apprivoiser progressivement des ambiances sonores nouvelles. Dans le premier cas, le projet Voix du tram suscite des émotions positives et amusées, permettant de réconforter une identité strasbourgeoise complexe, entre attachement au dialecte local et ouverture en tant que Capitale Européenne. Dans le projet Syneson, des ressentis globalement de rejet et d'impuissance autour de l'œuvre sont le signe (au-delà de l'aspect plus politique de l'affaire) d'une acculturation progressive à cette installation qui inscrit le Neudorf dans un espace symbolique gentrifié, par le lien entre gentrification et art contemporain. Dans le troisième cas, le SONar permet de rendre compte de l'acceptation de la transformation d'une place à Hautepierre, mais indique aussi que les transformations n'ont pas permis le changement d'image souhaité par la rénovation urbaine. Le sonore est donc ressenti différemment par les usagers et utilisé de manière variable par les projets : dans des espaces à enjeu urbain fort, comme le tram, ou des quartiers qui se gentrifient, comme le Neudorf, la municipalité emploie l'art public sonore afin de donner des repères, de déterminer des jugements, de faciliter l'identification des nouveaux lieux à travers des "signatures sonores ». Dans des quartiers périphériques comme Hautepierre, la rénovation urbaine ne prévoit pas d'art contemporain, encore moins sonore. Le travail de récolte des 
mémoires porté par Horizome permet alors l'existence d'une parole alternative.

Quels sont les relations entre ces lieux ? Loin de penser que ces projets s'opposent les uns aux autres, même si leur sens est très différent, des liens existent et parfois une démarche associative parvient à insuffler une vision participative dans la méthode de projets institutionnels ${ }^{1{ }^{1}}$. Dans tous les cas présentés, il s'agit d'admettre que l'art sonore participe à fabriquer l'identité des lieux ou à la révéler aux usagers. En conséquence, si "le son publicise l'espace » il est aussi le signe d'une certaine manière de concevoir la "ville aimable " (Martouzet, 2014) de la part des institutions et des aménageurs, en lien avec des changements urbains qu'ils ont souhaités.

\section{Bibliographie :}

ADEUS (201") Typologie des quartiers CUS, Strasbourg.

AMPHOUX P. (2014) « Environnement, milieu, paysage. Un modèle pour concevoir " Conférence, École d'hiver du CRESSON

www.cresson.archi.fr/ENS/winterSCHOOL/winterSCHOOL-2014.html, consulté le 0\%/08/15.

AUDAS N. (2010), " La dimension affective du rapport au lieu des individus : techniques d'enquêtes comparées», Natures Sciences Sociétés, 2, Vol. 18, pp. 195-201.

www.cairn.info/revue-natures-sciences-societes-2010-2-page-195.htm, consulté le 15/09/15.

AUGOYARD J-F. (1979) Pas à pas. Essai sur le cheminement quotidien en milieu urbain, Paris, Éditions du Seuil.

BAUTES N., GUIU C. (2010), "Cheminements autour de l'identité urbaine ", in Gerardot M., La France en ville, Paris, Éditions Atlande, pp.119-126.

BERNARD J. (2015), "Les voies d'approche des émotions. Enjeu de définition et catégorisations ", Terrains Théories, $\mathrm{n}^{\circ} 2$.

http://teth.revues.org/196, consulté le 0\%/01/16.

CHADOIN O. (2010), "La notion d'ambiance. Contribution à l'examen d'une invention intellectuelle post-moderne dans le monde de la recherche architecturale et urbaine ", Les Annales de la recherche urbaine, $\mathrm{n}^{\circ} 106$, pp. $15^{\prime \prime}-159$.

CRAPANZANO V. (1994), " Réflexions sur une anthropologie des émotions », Terrain, ²2, pp.109117.

DELAGE B. (1989) Paysage sonore urbain, Éditions Plan construction.

DESGRANDCHAMP P. (2014), "Le designer des sons de l'urbain, vers une écriture scénophonique de la ville » dans Bertrand G., et Favard M., Poïétiques du design, Vers de nouveaux paradigmes de la conception?, Paris, Éditions L'Harmattan, pp. 71-86.

DERNIERES NOUVELLES D'ALSACE (2001), " Les voix du tram », 19 Décembre.

DERNIERES NOUVELLES D'ALSACE (2012), " L'arbre sonore qui cache la forêt ", 2" Octobre.

DERNIERES NOUVELLES D'ALSACE (201“), « Un cœur à prendre », 1 décembre.

\footnotetext{
1" C'est la rencontre entre le Shadok pour la Ville de Strasbourg et les associations Av-lab et Horizome qui a permis la mise en place du projet FIP : fabriquer, inventer, partager : "Hautepierre à l'heure du numérique ", projet lauréat du Fonds d'expérimentation de la jeunesse du ministère de la Ville, la Jeunesse et des Sports par l'appel "Education populaire par et pour les jeunes : pratiques numériques, lieux innovants, médias de jeunes". Toutefois il est à noter que cet aspect collaboratif, l'écriture du projet à trois voix, fut limité par le cahier des charges même de l'appel qui ne prenait pas en compte plusieurs porteurs de projet : finalement, c'est bien le Shadok, structure institutionnelle et porteur du projet officiel, qui a été le seul lauréat.
} 
DERNIERES NOUVELLES D'ALSACE (2013), “ Opération pédagogie », 14 Juillet.

DERNIERES NOUVELLES D'ALSACE (2015), "Cinquante nuances de silence », 15 février.

DERNIERES NOUVELLES D'ALSACE (2015), « Un silence qui fait parler », 15 février.

FAUBERT J. (2012), "Corps critiques : parcours sonores urbains artistiques et espace public " dans Thibaud, J.-P. et Siret, D., Ambiances in action/Ambiances en acte(s) -International Congress on Ambiances, Montrel 2012, International Ambiances Network, pp. 429-434.

FEILDEL B. (2013), "Vers un urbanisme affectif. Pour une prise en compte de la dimension sensible en aménagement et en urbanisme $"$, Norois $n^{\circ} 227$, pp. 55-68.

FEILDEL B. (2010) Espaces et projets à l'épreuve des affects. Pour une reconnaissance du rapport affectif à l'espace dans les pratiques d'aménagement et d'urbanisme, Thèse de doctorat en aménagement de l'espace et urbanisme, Université F. Rabelais - Tours.

FRIAS A. (2001), "Une introduction à la ville sensible ", Recherches en anthropologie au Portugal n7, pp. 11-36.

GERVAIS-LAMBONY P. (2004), « De l'usage de la notion d'identité en géographie. Réflexions à partir d'exemples sud-africains », Annales de Géographie,113/638, pp. 469-488.

GUASTAVINO C. (2006), "The ideal urban soundscape : Investigating the sound quality of French cities ", Acta Acustica United with Acustica 92 (6), pp. 945-951

GUINARD P. (2010), «Quand l'art public (dé)fait la ville ? », EchoGéo, n¹3.

GUTTON J.P. (1970) Bruits et sons dans notre histoire, Paris, Éditions PUF.

HAMMAN P. (2011), "La négociation à l'épreuve des projets urbains de tramway ", in Hamman P., Le tramway dans la ville, Rennes, Presses universitaires de Rennes, pp. 7-42.

LEIPP E. (1977) La machine à écouter, essai de psycho-acoustique, Paris, Éditions Masson.

MARTOUZET D. (2007), «Le rapport affectif à la ville: positionnement théorique et épistémologique ", Praxis, Revue électronique d’Aménagement.

MARTOUZET D. (2013), " Introduction. Une ville, cinq sens, trois traitements : sensoriel, cognitif et affectif $"$, Norois $n^{\circ} 227$, pp. 7-10.

http://norois.revues.org/4635, consulté le 22/08/15.

MARTOUZET D. (2014) Ville aimable, Tours, Presses Universitaires François Rabelais.

MARRY S. (2011) L'espace public sonore ordinaire : les paramètres de la perception sonore dans les espaces publics : contribution à une connaissance de l'ambiance sonore, Thèse en architecture et aménagement de l'espace, Universite de Grenoble.

MAYR A. (2012), "Deux écologies pour les basses fréquences", Sonorité $n^{\circ} 7$, Ecologie sonore entre sens, art, science, pp. 32-35.

MOLES A. (1973) Théorie de l'information et perception esthétique, Paris, Éditions Denoël.

MOROVICH B. (2012), "Concertation et projet urbain à Hautepierre : la structuration d'une démocratie participative ", Revue des Sciences Sociales. La ville durable, pp. 64-73.

MOROVICH B. (2014), "Entre stigmates et mémoires: dynamiques paradoxales de la rénovation urbaine ", Articulo - Journal of Urban Research, Special issue 5.

http://articulo.revues.org/2529, consulté le 03/08/15.

MOROVICH B. (2015), "Médias, rénovation urbaine et associations artistiques : faiseurs d'images dans les quartiers populaires en France " in Juliet Carpenter J., Horvath C., Regards croisés sur la banlieue, Bruxelles, Peter Lang, pp. 57-87.

MURRAY SCHAFER R. (2010) Le paysage sonore, le monde comme musique, Paris, Éditions Wildproject. 
ORSELLI J. (2004) Les nouveaux tramways : comment ne pas rater son tram, Orléans, Paradigme. REDONDO B. (2012), "Tramway et territoire : quel urbain en perspective? ", Revue Géographique de l'Est, vol. 52 / 1-2.

SALADIN M. (2015) L'expérience de l'expérimentation, Dijon, Éditions les presses du réel.

SCHAEFFER P. (1966) Traité des objets musicaux, Paris, Éditions du Seuil.

TARDIEU J. (2006) De l'ambiance à l'information sonore dans un espace public, méthodologie et réalisations appliquées aux gares, Thèse en Acoustique, Université Paris VI.

TIBERGHIEN G.A. (2000), "Les voix du tram, rencontre entre Gilles A. Tiberghien et Rodolphe Burger ", Vacarmes n¹4, pp. 111-115.

http://www.vacarme.org/article90.html, consulté le 15/09/15.

VESCHAMBRE V. (2008) Traces et mémoires urbaines, enjeux sociaux de la patrimonialisation et de la démolition, Rennes, Éditions PUR.

VIVANT E. (2009) Qu'est-ce que la ville créative ?, Paris, PUF.

VOLCLER J. (2013), " le marketing sonore envahit les villes ", Le monde diplomatique :

http://www.monde-diplomatique.fr/2013/08/VOLCLER/49545\#nh4, consulté le 03/08/15.

VON UEXKULL J. (1965) Mondes animaux et monde humain, Paris, Éditions Denoël. 